\title{
Chimeric Monoclonal Antibody B72.3
}

National Cancer Institute

\section{Source}

National Cancer Institute. Chimeric Monoclonal Antibody B72.3. NCI Thesaurus. Code C28886.

A synthetic chimeric molecule in which a mouse monoclonal antibody variable region directed against the tumor-associated glycoprotein 72 (TAG-72) is fused to a human antibody constant region. This agent reacts to most human adenocarcinomas (colorectal, gastric, pancreatic, ovarian, endometrial, mammary) but has weak or undetectable reactivity to most normal tissues. ( $\mathrm{NCl04)}$ 